\title{
Minimization of Hydrocarbon Accumulation on Nanomanipulator Probe Tips
}

\author{
G. McMahon, * Christopher G.. Morgan, ${ }^{* *}$ and Ronald Vane** \\ * Nanofabrication Cleanroom Facility, Boston College, Newton, MA 02459 \\ ** XEI Scientific, Inc., Redwood City, CA 94063
}

Electrical characterization of nanodevices is out of necessity frequently performed in-situ within an SEM, dual beam or even a TEM with the aid of nanomanipulators. Although nanomanipulator technology is maturing and their use rapidly gaining popularity, there remain several areas where improvement in the reproducibility of measurement could be facilitated. One well known problem is that of establishing Ohmic contacts to the device to be measured [1]. For the case where tungsten probe tips are used, poor electrical contact can be a result of debris build-up on the tip, the presence of an oxide layer left over from the probe tip preparation method [2], and the focus of this study: Electron beam induced hydrocarbon layer accumulation.

In this work, we examine the effectiveness of downstream cleaning by oxygen radicals generated by a plasma device for the prevention of hydrocarbon buildup on clean tungsten probe tips.

The control measurements were performed in the following manner: New W probe tips (GGB Industries Inc., Naples FL) were removed from their Argon filled package, rinsed in distilled water, rinsed in isopropyl alcohol, and then blown dry with nitrogen. All four tips were installed into four Kleindiek MM3A micromanipulators within a JEOL 4500 Multibeam specimen chamber and the system evacuated to $1 \times 10^{-3} \mathrm{~Pa}$. Two probes were placed on either end of a $52 \Omega$ surface mount resistor and two point measurements were performed using a Keithley 2612A sourcemeter. The current was swept from $-100 \mu \mathrm{A}$ to $+100 \mu \mathrm{A}$ and the voltage drop measured and resistance calculated. After this measurement, the magnification on one probe was increased to $15,000 \mathrm{X}$, the probe lifted until it was no longer contacting the surface, and left under the beam for 15 minutes. After this time, the probe was replaced back on the surface, and the electrical properties measured again. The process was repeated several times to investigate the cumulative effect on the probe tip after several 15 minute increments under the beam at 15,000X.

A similar protocol was used to investigate the effect of the plasma cleaning treatment: All four probes were removed from the chamber, rinsed in distilled water, then in isopropyl alcohol, and finally blown dry with nitrogen and re-installed in the chamber. Pumping was initiated and the Evactron 25 De-Contaminator RF Plasma Cleaning System (XEI Scientific Inc., Redwood City, CA) was run for 20 minutes at a power of $14 \mathrm{~W}$ at a pressure of $53 \mathrm{~Pa}$. Following the plasma treatment, the Evactron then purged the chamber with dry nitrogen for 5 minutes at a pressure of $80 \mathrm{~Pa}$ before completing its cycle and allowing the dual beam instrument to continue pumping until a vacuum of 1 $\mathrm{x} 10^{-3} \mathrm{~Pa}$ was obtained. The probes were then set up in the same manner as before, and the exact same procedure was used (15 minute intervals, $15,000 \mathrm{X}, 15 \mathrm{kV}$ and the same spot size). Although it was not possible to measure the SEM beam current directly, the spot size setting and lens current in the $\mathrm{LaB}_{6}$ source were identical for all measurements.

The results of the resistance measurements from the control and the Evactron cleaning experiments are shown in Table 1. 
TABLE 1: Resistance Measurements from Control and Evactron Cleaning Experiments

\begin{tabular}{|c|c|c|}
\hline Cumulative Time Under SEM & \multicolumn{2}{|c|}{ Measured Resistance $(\Omega)$} \\
\cline { 2 - 3 } $\begin{array}{c}\text { Electron Beam at 15kX } \\
\text { magnification (minutes) }\end{array}$ & Control & Evactron Cleaning \\
\hline 15 & 55 & 55 \\
\hline 30 & 56 & 56 \\
\hline 45 & 64 & 58 \\
\hline 60 & $\begin{array}{c}11,600 \\
\text { Tip sputter cleaned with FIB } \\
\text { from one side }\end{array}$ & 60 \\
\hline 75 & 55 & 68 \\
\hline 90 & 56 & 81 \\
\hline 105 & 2900 & $\begin{array}{c}\text { Acquire FIB image (no } \\
\text { focusing, 1 raster pass) }\end{array}$ \\
\hline 120 & 7850 & 59 \\
\hline & & 62 \\
\hline 135 & 14,000 & \\
\hline
\end{tabular}

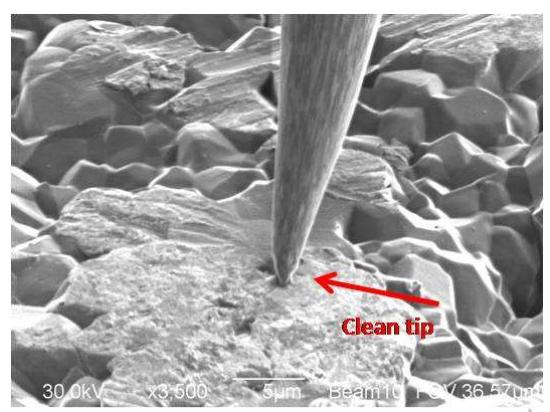

FIG. 1. Clean probe tip contacting resistor surface at beginning of experiment.

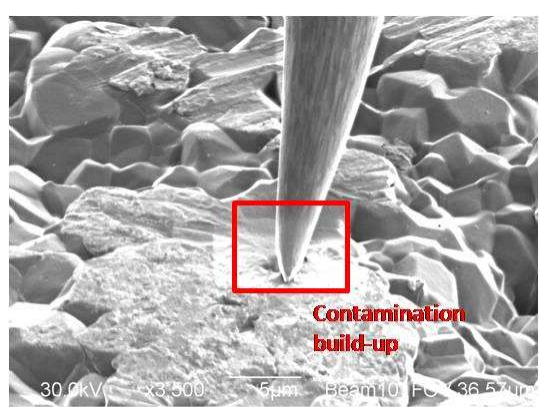

FIG. 2. Control experiment. Probe tip after 45 minutes under the SEM beam at $15,000 \mathrm{X}$.

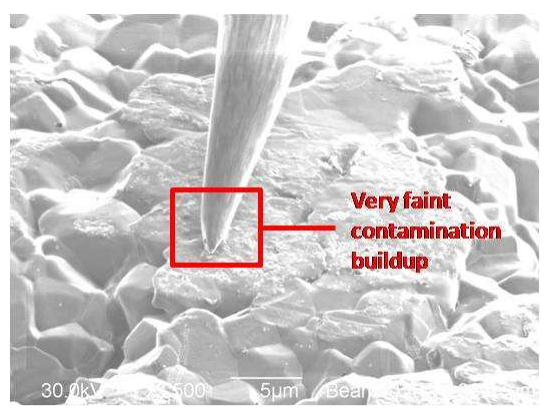

FIG. 3. Evactron cleaning experiment. Probe tip after 120 minutes under the SEM beam at $15,000 \mathrm{X}$.

A positive effect of downstream plasma cleaning by oxygen radicals generated from the Evactron plasma cleaning system is evident. In the control experiment, exposure of the tungsten probe tips to the beam at $15 \mathrm{kX}$ magnification for only 60 minutes led to very high resistance measurements (Table 1) and an obvious accumulation of hydrocarbons on the probe tip (Fig. 1 and 2). The same measurements performed after Evactron cleaning were much more reliable, even after 75 minutes under the beam, and the large increases in resistance and obvious hydrocarbon buildup on the probe tip were not observed (Table 1 and Fig. 3). We have also found that after further cleaning of the chamber over time, the slight increases in resistance we observe after Evactron cleaning subsided.

References

[1] Y. Peng et al., Journal of Physics: Conference Series 126 (2008) 012031.

[2] Z. Saghi et al., Appl. Phys. Lett. 91 (2007) 251906. 